\title{
Varying Light Velocity as a Solution to the Problems in Cosmology
}

\author{
J. W. Moffat \\ Department of Physics, University of Toronto, Toronto, Ontario M5S 1A7, \\ Canada
}

\begin{abstract}
In earlier published work, it was proposed that light speed was larger in the early universe by 30 orders of magnitude compared to its presently observed value. This change in the speed of light is associated with a spontaneous breaking of local Lorentz invariance in the early Universe, associated with a first order phase transition at a critical time $t=t_{c}$. This solves the horizon problem, leads to a mechanism of monopole suppression in cosmology and can resolve the flatness problem. it also offers the potential of solving the cosmological constant problem. After the critical time $t_{c}$, local Lorentz invariance is restored and light travels at its presently measured speed. We investigate the field equations in the spontaneously broken phase and study further the flatness problem and the cosmological constant problem. The entropy is shown to undergo a large increase as the light velocity goes through a phase transition. A scale invariant prediction for microwave background fluctuations originating at the time of the phase transition is discussed.
\end{abstract}

UTPT-98. e-mail: moffat@medb.physics.utoronto.ca

\section{Introduction}

The idea that the velocity of light varies in the early Universe and several of its consequences for cosmology was published some time ago[1, 2, [3]. The idea originated with the hypothesis that there is a phase of spontaneously broken, local Lorentz invariance and diffeomorphism invariance due to a nonvanishing vacuum expectation value (vev) of a field, $\phi$, shortly after the beginning of the Universe[3]. The local Lorentz and diffeomorphism symmetries 
of Einstein's gravitational theory are spontaneously broken by the symmetry breaking pattern: $S O(3,1) \rightarrow O(3)$ at a critical temperature $T_{c}$, below which the symmetry is restored. It was shown that this new scenario is capable of solving the horizon problem, the excess relic particle and flatness problems and leads to predictions for small scale inhomogeneities.

Recently, a series of papers, beginning with the paper by Albrecht and Magueijo[4-6], has appeared in which the velocity of light (and possibly the gravitational constant $G$ and other fundamental constants) was postulated to vary in the early universe.

In the following, we shall investigate further the physical consequences of the spontaneous breaking of the symmetries of spacetime in the early Universe, and the predictions for cosmology when the velocity of light goes through a first order phase transition at a critical time $t \sim t_{c}$.

\section{Spontaneous Breaking of Spacetime Sym- metries}

In the earlier work[1-3], we assumed that local Lorentz vacuum symmetry is spontaneously broken by a Higgs mechanism. We postulated the existence of four scalar fields, $\phi_{a}$, where $a$ labels the flat tangent space coordinates, and assumed that the vev of the scalar fields, $\left\langle\phi_{a}>_{0}\right.$, vanishes for some temperature $\mathrm{T}$ less than a critical temperature $T_{c}$, when the local Lorentz symmetry is restored. Above $T_{c}$ the non-zero vev will break the symmetry of the gound state of the Universe from $S O(3,1)$ down to $O(3)$. The domain formed by the direction of the vev of the field $\phi$ will produce an arrow of time pointing in the direction of increasing entropy and the expansion of the Universe.

Let us introduce the four real fields $\phi^{a}(x)(\mathrm{a}=0,1, \ldots 3)$, where $a, b, .$. denote flat tangent space coordinates. The fields $\phi^{a}$ are scalars in coordinate space with the coordinates $x^{\mu}$, and they are invariant under Lorentz transformations

$$
\phi^{\prime a}(x)=L_{b}^{a}(x) \phi^{b}(x) .
$$

We can use a vierbein $e^{a}{ }_{\mu}$ to convert $\phi^{a}$ into a 4 -vector in coordinate space: $\phi^{\mu}=e^{\mu}{ }_{a} \phi^{a}$. The $e_{\mu}^{a}$ satisfy

$$
e_{\mu}^{a} e_{b}^{\mu}=\delta_{b}^{a}, \quad e_{a}^{\mu} e_{\nu}^{a}=\delta_{\nu}^{\mu},
$$


and they obey the Lorentz transformation rule

$$
e_{\mu}^{\prime a}(x)=L_{b}^{a}(x) e_{\mu}^{b}(x) .
$$

The covariant derivative operator acting on $\phi^{a}$ is defined by

$$
D_{\mu} \phi^{a}=\left[\partial_{\mu} \delta_{b}^{a}+\left(\Omega_{\mu}\right)_{b}^{a}\right] \phi^{b},
$$

where $\left(\Omega_{\mu}\right)_{b}^{a}$ denotes the spin, gauge connection.

Consider the infinitesimal Lorentz transformation

$$
L_{b}^{a}(x)=\delta_{b}^{a}+\omega_{b}^{a}(x)
$$

with

$$
\omega_{a b}(x)=-\omega_{b a}(x) .
$$

The matrix $D(L)$ in the transformation rule for a general field $f_{n}(x)$ :

$$
f_{n}(x) \rightarrow \sum_{m}[D(L)(x)]_{n m} f_{m}(x)
$$

takes the form

$$
D[1+\omega(x)]=1+\frac{1}{2} \omega^{a b}(x) \sigma_{a b},
$$

where the $\sigma_{a b}$ are the six generators of the Lorentz group which satisfy $\sigma_{a b}=$ $-\sigma_{b a}$ and the commutation rules

$$
\left[\sigma_{a b}, \sigma_{c d}\right]=\eta_{c b} \sigma_{a d}-\eta_{c a} \sigma_{b d}+\eta_{d b} \sigma_{c a}-\eta_{d a} \sigma_{c b} .
$$

The set of scalar fields $\phi$ transforms as

$$
\phi^{\prime}(x)=\phi(x)+\omega^{a b}(x) \sigma_{a b} \phi(x) .
$$

The gauge spin connection which satisfies the transformation law

$$
\left(\Omega_{\sigma}\right)_{b}^{a} \rightarrow\left[L \Omega_{\sigma} L^{-1}-\left(\partial_{\sigma} L\right) L^{-1}\right]_{b}^{a},
$$

is given by

$$
\Omega_{\mu}=\frac{1}{2} \sigma^{a b} e_{a}^{\nu} e_{b \nu ; \mu},
$$

where ; denotes covariant differentiation with respect to the Christoffel symbol $\Gamma_{\mu \nu}^{\lambda}$ :

$$
\Gamma_{\mu \nu}^{\lambda}=g^{\lambda \rho} \eta_{a b}\left(D_{\mu} e_{\nu}^{a}\right) e_{\rho}^{b} .
$$


A Higgs sector is included in the Lagrangian density such that the gravitational vacuum symmetry, which is set equal to the Lagrangian symmetry at low temperatures, breaks to a smaller symmetry at high temperature. The pattern of vacuum phase transition that emerges contains a symmetry anti-restoration. This vacuum symmetry breaking leads to the interesting possibility that exact zero temperature conservation laws e.g. electric charge and baryon number are broken in the early Universe. It was shown that the spontaneous breaking of the Lorentz symmetry of the vacuum leads to a violation of the exact zero temperature conservation of energy in the early Universe, which can explain the origin of matter in the big bang.

Let us consider the Lorentz invariant potential:

$$
V(\phi)=-\frac{1}{2} \mu^{2} \sum_{a=0}^{3} \phi_{a} \phi^{a}+\lambda \sum_{a=0}^{3}\left(\phi_{a} \phi^{a}\right)^{2},
$$

where we choose $\phi^{a}$ to be a timelike Lorentz vector, $\phi_{a} \phi^{a}>0$, and $\lambda>0$, so that the potential is bounded from below. If $V$ has a minimum at $\phi_{a}=$ $v_{a}$, then the spontaneously broken solution is given by $v_{a}^{2}=\mu^{2} / 4 \lambda$ and an expansion of $V$ around the minimum yields the mass matrix:

$$
\left(\mu^{2}\right)_{a b}=\frac{1}{2}\left(\frac{\partial^{2} V}{\partial \phi_{a} \partial \phi_{b}}\right)_{\phi_{a}=v_{a}} .
$$

We can choose $\phi_{a}$ to be of the form

$$
\phi_{a}=\left(\begin{array}{l}
0 \\
0 \\
0 \\
v
\end{array}\right)=\delta_{a 0}\left(\mu^{2} / 4 \lambda\right)^{1 / 2}
$$

All the other solutions of $\phi_{a}$ are related to this one by a Lorentz transformation. Then, the homogeneous Lorentz group $S O(3,1)$ is broken down to the spatial rotation group $O(3)$. The three rotation generators $J_{i}(i=1,2,3)$ leave the vacuum invariant

$$
J_{i} v_{i}=0
$$

while the three Lorentz-boost generators $K_{i}$ break the vacuum symmetry

$$
K_{i} v_{i} \neq 0
$$


The mass matrix $\left(\mu^{2}\right)_{a b}$ can be calculated from (15):

$$
\left(\mu^{2}\right)_{a b}=\left(-\frac{1}{2} \mu^{2}+2 \lambda v^{2}\right) \delta_{a b}+4 \lambda v_{a} v_{b}=\mu^{2} \delta_{a 0} \delta_{b 0},
$$

where $v$ denotes the magnitude of $v_{a}$. There are three zero-mass Goldstone bosons, the same as the number of massive vector bosons, $V_{\mu}^{i}=$ $\left(\Omega_{\mu}\right)_{0 i}=-\left(\Omega_{\mu}\right)_{i 0}$, and there are three massless vector bosons, $U_{\mu}^{n}=\left(\Omega_{\mu}\right)_{m n}=$ $-\left(\Omega_{\mu}\right)_{n m}$, corresponding to the unbroken $O(3)$ symmetry. In addition to these particles, one massive physical boson particle $h$ remains, after the spontaneous breaking of the vacuum.

A phase transition is assumed to occur at the critical temperature $T_{c}$, when $v_{a} \neq 0$ and the Lorentz symmetry is broken and the three gauge fields $\left(\Omega_{\mu}\right)_{i 0}$ become massive vector bosons. Below $T_{c}$ the Lorentz symmetry is restored, and we regain the usual classical gravitational field with massless gauge fields $\Omega_{\mu}$. The symmetry breaking will extend to the singularity or the possible singularity-free initial state of the big bang, and since quantum effects associated with gravity do not become important before $T \sim 10^{19}$ $\mathrm{GeV}$, we expect that $T_{c} \leq 10^{19} \mathrm{GeV}$.

The total action for the theory is

$$
S=S_{G}+S_{M}+S_{\phi},
$$

where the action for Einstein gravity is

$$
S_{G}=-\frac{c^{4}}{16 \pi G} \int d^{4} x e(R+2 \Lambda) .
$$

$e \equiv \sqrt{-g}=\operatorname{det}\left(e_{\mu}^{a} e_{a \nu}\right)^{1 / 2}, \Lambda$ is the cosmological constant and $S_{M}$ is the matter action for gravity. Moreover,

$$
S_{\phi}=\int d^{4} x \sqrt{-g}\left[\frac{1}{2}\left(D_{\mu} \phi_{a} D^{\mu} \phi^{a}-V(\phi)\right] .\right.
$$

By choosing $\phi^{a}$ to be a Lorentz timelike vector, we ensure that the kinetic energy term $D_{\mu} \phi_{a} D^{\mu} \phi^{a}>0$ for all events in the past and future light cones of the flat tangent space. Since the kinetic energy term is positive definite within the light cone and $\phi_{a} \phi^{a}>0$, we guarantee that local Lorentz invariance is broken within the past and future light cones where spacetime events are causally connected. 
Let us consider small oscillations about the true minimum and define a shifted field

$$
\phi_{a}^{\prime}=\phi_{a}-v_{a} .
$$

We perform a Lorentz transformation on $\phi^{a}$, so that we obtain

$$
\phi^{0}=h, \quad \phi^{1}=\phi^{2}=\phi^{3}=0 .
$$

In this special coordinate frame, the remaining component $h$ is the physical Higgs particle that survives after the three Goldstone modes have been removed. This corresponds to choosing the "unitary gauge" in the standard electroweak model.

The total action for the theory in the broken symmetry phase, $T>T_{c}$, is

$$
S=S_{G}+S_{M}+S_{h}+S_{V}
$$

In our specially chosen coordinate frame in which (24) holds, we have

$$
S_{h}=\int d^{4} x \sqrt{-g}\left[\frac{1}{2} \partial_{\mu} h \partial^{\mu} h-V(h)\right],
$$

where

$$
V(h)=4 \lambda v^{2} h^{2}+4 \lambda v h^{3}+\lambda h^{4}-\frac{1}{2} V_{\mu}^{2} h^{2}-v V_{\mu}^{2} h,
$$

and we have for convenience suppressed the index $i$ on $V_{\mu}^{i}$. Moreover,

$$
S_{V}=\frac{1}{2} m^{2} \int d^{4} x \sqrt{-g} g^{\mu \nu} V_{\mu} V_{\nu},
$$

where the mass $m \propto<h>_{0}$.

The field equations are of the form:

$$
G^{\mu \nu} \equiv R^{\mu \nu}-\frac{1}{2} g^{\mu \nu} R=\frac{8 \pi G}{c^{4}}\left(T^{\mu \nu}+K^{\mu \nu}+H^{\mu \nu}\right)+\Lambda g^{\mu \nu},
$$

where $K^{\mu \nu}$ is given by

$$
K^{\mu \nu}=m^{2}\left(V^{\mu} V^{\nu}-\frac{1}{2} g^{\mu \nu} V^{\beta} V_{\beta}\right) .
$$

Moreover, the $h$ field energy-momentum tensor is of the usual form:

$$
H^{\mu \nu}=\partial^{\mu} h \partial^{\nu} h-\mathcal{L}_{h} g^{\mu \nu} .
$$


Since $G^{\mu \nu}$ satisfies the Bianchi identities:

$$
G_{; \nu}^{\mu \nu}=0
$$

we find from $(29)$ that

$$
T_{; \nu}^{\mu \nu}=-\left(K^{\mu \nu}+H^{\mu \nu}\right)_{; \nu},
$$

where we have used $g_{: \nu}^{\mu \nu}=0$. In the unbroken phase of spacetime, we regain the standard energy-momentum conservation laws $\left(K^{\mu \nu}=0, H_{; \nu}^{\mu \nu}=0\right)$ :

$$
T_{; \nu}^{\mu \nu}=0
$$

and the spin connection corresponds to a massless graviton gauge field.

\section{Field Equations in the Broken Symmetry Phase}

The spacetime manifold in the broken phase has the symmetry $R \times O(3)$. The three-dimensional space with $O(3)$ symmetry is assumed to be homogeneous and isotropic and yields the usual maximally symmetric three-dimensional space:

$$
d \sigma^{2}=R^{2}(t)\left[\frac{d r^{2}}{1-k r^{2}}+r^{2}\left(d \theta^{2}+\sin ^{2} \theta d \phi^{2}\right)\right],
$$

where $t$ is the external time variable. This is the Robertson-Walker theorem for our ordered phase of the vacuum and it has the correct subspace structure for the FRW Universe with the metric:

$$
d s^{2}=d t^{2} c^{2}-R^{2}(t)\left[\frac{d r^{2}}{1-k r^{2}}+r^{2}\left(d \theta^{2}+\sin ^{2} \theta d \phi^{2}\right)\right] .
$$

In the broken symmetry phase, the "time" $t$ is the absolute physical time measured by standard clocks. In contrast to GR, while $\left\langle\phi>_{0}\right.$ is non-zero, we no longer have re-parameterization invariance and time is no longer an arbitrary label.

Let us consider Einstein's field equations in the broken symmetry phase $T>T_{c}$. We have $g_{00}(t)=c_{0}^{2}$ and $g_{i k}(t)=-R^{2}(t) \gamma_{i k}$ and the energymomentum tensor for a perfect fluid takes the form:

$$
T^{\mu \nu}=\left(\rho+\frac{p}{c_{0}^{2}}\right) u^{\mu} u^{\nu}-p g^{\mu \nu},
$$


where $c_{0}$ labels the velocity of light in this epoch, $u^{\mu}=d x^{\mu} / d s, u^{\mu} u_{\mu}=$ $c_{0}^{2}, \rho$ is the density of matter and radiation and $p$ is the pressure. In our homogeneous space the spatial part of the massive vector field $V^{i}=0$. Let

us use the notation: $V^{0}(t)=\chi(t)$. We obtain the field equations in the broken symmetry phase

$$
\begin{gathered}
\frac{\dot{R}^{2}}{c_{0}^{2} R^{2}}+\frac{k}{R^{2}}=\frac{8 \pi G}{3 c_{0}^{2}} \rho+\frac{8 \pi G}{c_{0}^{4}}\left[p+\frac{1}{2} m^{2} \chi^{2}+\frac{1}{2}(\dot{h})^{2}+V(h)\right]+\frac{\Lambda}{3}, \\
2\left(\frac{\ddot{R}}{c_{0}^{2} R}\right)+\frac{\dot{R}^{2}}{c_{0}^{2} R^{2}}+\frac{k}{R^{2}}=-\frac{8 \pi G}{c_{0}^{4}}\left[p+\frac{1}{2} m^{2} \chi^{2}+\frac{1}{2}(\dot{h})^{2}-V(h)\right]+\Lambda, \\
\frac{1}{R^{3}} \frac{\partial}{\partial t}\left[R^{3} c_{0}^{2}\left(\rho+\frac{p}{c_{0}^{2}}\right)\right]-\dot{p}=-W,
\end{gathered}
$$

where $\dot{R}=d R / d t$ and $W$ is given by

$$
W=m^{2} \chi\left[\dot{\chi}+3\left(\frac{\dot{R}}{R}\right) \chi\right]+(\dot{h})^{2}\left[1+3\left(\frac{\dot{R}}{R}\right)\right]+V^{\prime}(h) \dot{h} .
$$

\section{Superluminary Universe}

The horizon scale is determined by

$$
d_{H}(t)=c_{0} R(t) \int_{0}^{t} \frac{d t^{\prime}}{R\left(t^{\prime}\right)}
$$

For $t>t_{c}$, this will have the usual value: $d_{H}(t)=2 c t$, since $R(t) \propto t^{1 / 2}$ for a radiation dominated Universe. Let us assume that for $t \leq t_{c}$, the speed of light is very large. During a first order phase transition, the velocity of light is assumed to undergo a discontinuous change from the value:

$$
c_{0} \sim a c
$$

for $t \leq t_{c}$ to $c_{0}=c$ (c is the present value of the velocity of light and $a$ is a constant) for $t>t_{c}$. Then, we get for $t \leq t_{c}$ :

$$
d_{H}(t) \approx c_{0} g(t)
$$

where $g(t)$ is the dynamical time dependence arising from $R(t)$ in (42). Thus, for a fraction of time near the beginning of the Universe, and for $a \rightarrow \infty$, 
all points of the expanding space will have been in communication with one another solving the horizon problem.

Suppose the field $\phi$ that breaks the spacetime symmetries is characterized by a correlation length $\xi$. Then, the monopole density is approximately given by

$$
n_{M} \approx \xi^{-3}
$$

In the superluminary model, the bound on the length $\xi$ is given by

$$
\xi<d_{H}(t) \approx c_{0} g(t)
$$

so that the bound on the number density of monopoles is exponentially weakened. This solves the relic particle (monopole) problem.

The present observational data restrict $\Omega_{0}=\rho_{\text {crit }} / \rho_{0}$ to lie in the interval $[0.01$, few $]$, which implies that $R_{\text {curv }} \sim c / H_{0}$ and $\rho_{0} \sim \rho_{\text {crit }}$. From Eq.(38) in the broken phase, we can derive the expression

$$
\Omega(t)=1 /[1-x(t)]
$$

where in the radiation dominated superluminary era

$$
x(t)=\frac{c_{0}^{2} k}{R^{2} H^{2}} \sim \frac{c_{0}^{2} k / R^{2}}{8 \pi G \rho_{r} / 3},
$$

where $\rho_{r}$ denotes the radiation density. Moreover we have

$$
\Omega=\Omega_{\rho_{r}}+\Omega_{F}+\Omega_{\Lambda}
$$

where

$$
\begin{gathered}
\Omega_{\rho_{r}}=\frac{8 \pi G \rho_{r}}{3 H^{2}}, \quad \Omega_{F}=\left(\frac{8 \pi G}{c_{0}^{2} H^{2}}\right)\left[p+\frac{1}{2} m^{2} \chi^{2}+\frac{1}{2}(\dot{h})^{2}+V(h)\right], \\
\Omega_{\Lambda}=\frac{c_{0}^{2} \Lambda}{3 H^{2}} .
\end{gathered}
$$

We have in the radiation dominated era, $\rho_{r}=\rho_{0 r}\left(R_{0} / R\right)^{4}$, so that

$$
x \sim \frac{c_{0}^{2} k R^{2}}{R^{*}}
$$


where $R^{*}=8 \pi G \rho_{0 r} R_{0}^{4} / 3$. This yields

$$
\left|\Omega\left(10^{-43} \mathrm{sec}\right)-1\right| \sim O\left(a^{2} 10^{-60}\right) .
$$

Thus, in the short time that the Universe is superluminary with $a \sim 5 \times 10^{29}$, we get

$$
\left|\Omega\left(10^{-43} \mathrm{sec}\right)-1\right| \sim O(1),
$$

which implies much less fine tuning than the standard FRW model.

We observe that in contrast to the inflationary model (which predicts that $\Omega=1$ ) [12, 13], the prediction for the value of $\Omega$ in the superluminary model depends on the detailed dynamics of the theory [1, 田, 5, 60. Indeed, if we were to assume the equation of state: $\rho=$ const., that $\chi$ and $V$ are uniform in the broken phase and $k=0$, then $R(t)$ has the inflationary solution:

$$
R(t) \propto \exp \left[t c_{0}(\Lambda / 3)^{1 / 2}\right] .
$$

Thus we would regain the standard inflationary prediction $\Omega=1$. Clearly, the superluminary model does not lead automatically to the generic prediction $\Omega=1$. The possibility of obtaining an open universe version of the inflationary scenario has been the subject of much controversy recently [7, 8, 9]. The fact that obtaining an open Universe in the superluminary model is not a problem is a positive feature in favour of the model.

Let us now consider the cosmological constant problem. We ignore the effects of the $h$ and $V$ fields, since they will not play an important role in the present discussion. Then, using the equation of state: $p=\frac{1}{3} \rho$, we can derive at some instant of time $\bar{t}$ :

$$
H^{2}(\bar{t})(q(\bar{t})-1)=\frac{c_{0}^{2} k}{R^{2}(\bar{t})}-\frac{2 \Lambda c_{0}^{2}}{3},
$$

where $q$ is the deceleration parameter

$$
q=-\ddot{R} R / \dot{R}^{2} .
$$

We also have

$$
\frac{c_{0}^{2} k}{R^{2}(\bar{t}) H^{2}(\bar{t})}=\Omega(\bar{t})-1+\frac{c_{0}^{2} \Lambda}{3 H^{2}(\bar{t})} .
$$

It follows that

$$
|q(\bar{t})-\Omega(\bar{t})|=\left|\frac{c_{0}^{2} \Lambda}{3 H^{2}(\bar{t})}\right| .
$$


Assuming that the radiation dominant solution of the field equations holds near the phase transition, then $\Omega(\bar{t}) \sim 0.1-1, q(\bar{t}) \sim 1, H(\bar{t}) \sim 1 / 2 \bar{t}$ and

$$
R(\bar{t})=\left(\frac{32 \pi G \rho_{0 r}}{3}\right)^{1 / 4} R_{0} \bar{t}^{1 / 2}
$$

We obtain

$$
|\Lambda| \approx \frac{1}{c_{0}^{2} \bar{t}^{2}}
$$

For a rapid phase transition in the velocity of light and for $c_{0}=a c$ and $\log _{10} a \geq 60$, we have for $\bar{t} \sim 10^{-43}$ sec:

$$
|\Lambda|<10^{-54} \mathrm{~cm}^{-2} \text {. }
$$

From the critical density $\rho_{\text {crit }} \sim \rho_{0}$ this bound corresponds to $\Lambda / 8 \pi G \leq$ $8 \times 10^{-47} h^{2} \mathrm{GeV}^{4}$, where $0.4 \leq h \leq 1$. This would solve the cosmological constant problem[4, 5, 2]. The observational bound in (62) is obtained by using $H_{0} \sim 100 \mathrm{~km} \mathrm{~s}^{-1} \mathrm{Mpc}^{-1}$.

However, there is a serious conflict between the value $\log _{10} a \leq 30$ required to solve the flatness problem and the value $\log _{10} a>60$ required to solve the cosmological constant problem. Indeed, the latter value is far too large to accomodate a reasonable evolution of the FRW Universe just after the occurrence of the phase transition in the velocity of light. However, if we assume that another phase transition in the velocity of light occurs, before the one that solves the flatness problem, with $\log _{10} \sim 60$, then this could solve the cosmological constant problem and be followed by a phase transition with a lowering of the speed of light to a value with $\log _{10} \sim 30$, which could solve the flatness problem and allow the universe to expand to its present day value.

We have assumed the radiation dominant solution for $R(t)$ in this derivation. Perhaps another dynamical solution of the field equations would accomodate a solution to the horizon, flatness and cosmological constant problems in the presence of a phase transition in the velocity of light. Barrow [5] has considered the time dependent solutions of Brans-Jordan-Dicke theories with a field $\psi=c^{4}$, but such theories are severely restricted dynamically and can easily lead to consistency problems. In any eventuality, the horizon, flatness and monopole problems can be resolved by the superluminary model. The problem with the cosmological constant is not resolved in inflationary models, and indeed is exacerbated by the enormous vacuum energy density 
required to drive the initial inflation. The potential for the superluminary model to solve the cosmological constant problem could provide the model with another significant advantage.

One important aspect of the superluminary model is that it is not sensitive to the choice of an equation of state. No exotic forms of matter withe negative pressure are required to resolve cosmological problems. This is an advantage over the standard inflationary scenarios which require vacuum energy with $p=-\rho$ and unusual forms of potentials for the inflaton field that often require fine-tuning to implement the inflationary period.

\section{Black-Body Radiation and Entropy in the Superluminary Phase}

In the Lorentz symmetry broken phase of the Universe, the total blackbody radiation energy at temperature $T$ is given by

$$
u=\left(\frac{8 \pi h \nu^{3}}{c_{0}^{3}}\right) \int_{0}^{\infty}\left[\exp \left(\frac{h \nu}{k T}\right)-1\right]^{-1} d \nu=\frac{8 \pi^{5}(k T)^{4}}{15 h^{3} c_{0}^{3}} .
$$

The number density of photons is

$$
n_{\gamma}=\frac{60.42198(k T)^{3}}{\left(h c_{0}\right)^{3}} .
$$

The energy densities for photons and neutrinos are

$$
\epsilon_{\gamma}=\sigma_{B} T^{4}, \quad \epsilon_{\nu}=\frac{7}{16} \sigma_{B} T^{4},
$$

where $\sigma_{B}$ is the Stefan-Boltzmann constant

$$
\sigma_{B}=\frac{\pi^{2} k_{B}^{4}}{60 \hbar^{3} c_{0}^{3}}
$$

and $k_{B}$ is Boltzmann's constant. For $\log _{10} a \leq 30$ Stefan-Boltzmann's constant is $\sigma_{B} \sim 1.7 \times 10^{-84} \mathrm{erg} \mathrm{cm}^{-3} \mathrm{~K}^{-4}$ in the superluminary phase.

The Universe has negligible thermal and neutrino energy during the short period of the spontaneously broken symmetry phase. After the superluminary phase ends when $c_{0}=c$, both $\epsilon_{\gamma}$ and $\epsilon_{\nu}$ regain their standard values in 
an FRW Universe. In contrast to the inflationary model, there is no problem with a reheating epoch necessary in the inflationary scenario to replenish the matter and radiation in an 'empty' de-Sitter Universe. The velocity of light phase transition in the spontaneous symmetry breaking process automatically takes care of the creation of matter when $c_{0}=c[10,11]$.

The entropy of thermal photons is

$$
S(T)=\frac{4 \pi^{2} V}{45}\left(\frac{T}{\hbar c_{0}}\right)^{3} .
$$

Thus, at the phase transition at $t \sim t_{c}$, the entropy increases enormously in the direction of the expanding universe when $c_{0}=c$. The arrow of time connected with the increase of the entropy is determined by the domain arrow produced by the non-zero $\langle\phi\rangle_{0}$ in the spontaneously broken phase [1-3].

We see that the large value of $c$ in the early Universe, in the broken symmetry phase, changes radically the thermal physics at the beginning of the universe. This is in accord with the known observation that the entropy of a system increases rapidly as the system undergoes a first order or second order phase transition from an ordered to a more disordered state.

\section{Quantum Fluctuations and Density Pertur- bations}

Let us consider the possibility in our model of generating the seed perturbations that can grow to form the large-scale structures. During the superluminary phase for $t<t_{c}$, the fluctuation wavelengths grow as $\lambda \propto R(t)$. However, the horizon grows rapidly, $d_{H} \approx c_{0} g(t)$, where $c_{0}$ is given by (32), and it will become equal to the physical wavelength at some time $t=t_{\text {exit }}$, after which it becomes larger than $\lambda(k)$ for a mode labeled by a wave vector $\vec{k}$.

After the symmetry is restored at $t>t_{c}$, the proper length $R(t)$ grows as $t^{1 / 2}$, whereas the horizon will increase as $c H(t)^{-1} \sim c t$. Therefore, the wavelength will be completely within the Hubble radius for an interval of time $\Delta t$. Thus, in the superluminary model the fluctuations are in microcausal connection very early in the Universe $\left(t \sim 10^{-35} \mathrm{~s}\right)$ and have time to grow into physical modes sufficiently large to form Galaxy structures. These fluctuations will have a gaussian form, provided any self-couplings of the matter fields are small. 
The fluctuations associated with the Higgs field, $h$, could be a candidate for seed perturbations. The $h$ field satisfies

$$
\ddot{h}+3 H \dot{h}+V^{\prime}(h)=0,
$$

where $V^{\prime}(h)=d V(h) / d h$.

When the velocity of light undergoes a discontinuous change, during a first order phase transition at $t \sim t_{c}$, to the value $c_{0}$ given by (43) with $a \geq 10^{30}$, then the horizon, $d_{H}(t)$, determined by (42) will also have a discontinuity in its first derivative with respect to $t$, and $d_{H}(t)$ for $t<t_{c}$ can be matched to $d_{H}(t)$ for $t \geq t_{c}$ in such a way that $\lambda$ crosses $d_{H}(t)$ twice. The fluctuations are "frozen in" and leave an imprint on the metric tensor.

Fluctuations in $h$ give rise to perturbations in the density

$$
\delta \rho_{h}=\delta h\left(\frac{\partial V}{\partial h}\right) .
$$

At horizon crossings, $\lambda_{\text {phys }} \sim c H^{-1}$, the gauge invariant quantity $\zeta$ takes the simple form $\zeta=\delta \rho /\left(\rho+p / c^{2}\right)$ [14]. In the radiation dominated era and in the matter dominated era, $\zeta$ at horizon crossing is, up to a factor of order unity, equal to $\delta \rho / \rho$. Equating the values of $\zeta$ at the two horizon crossings, we find

$$
\left(\frac{\delta \rho}{\rho}\right)_{\text {Hor }} \sim \frac{\delta h V^{\prime}}{\dot{h}^{2}} \sim \frac{H V^{\prime}}{2 \pi \dot{h}^{2}},
$$

where we have used the fact that $\delta h \sim H / 2 \pi$. We must now model $V^{\prime}$ and $\dot{h}$ at the phase transition, in order to estimate the density fluctuation, $\delta \rho / \rho$. Clearly, $H$ is rapidly varying at the phase transition. We have

$$
\dot{h} \propto \frac{H}{2 \pi \delta t} .
$$

A natural time scale for the duration of the phase transition is given by

$$
\delta t \propto\left(\frac{H}{2 \pi h^{3}}\right)^{1 / 2} .
$$

Thus, if we choose $1 / H \sim 10^{-34} \mathrm{~s}$ and $h \sim M_{P} \propto 10^{43} s^{-1}$, then the duration of the phase transition is $\delta t \sim 10^{-48} \mathrm{~s}$.

By assuming that $V(h)$ is dominated by $V(h) \sim \frac{\lambda}{4} h^{4}$, we obtain from Eqs. (70), (71) and (72) the scale invariant prediction for the amplitude

$$
\left(\frac{\delta \rho}{\rho}\right)_{\text {Hor }} \sim \lambda
$$


We can fit the data measured by the Cosmic Background Explorer (COBE), which is consistent with a gaussian, scale invariant spectrum[15, 16], by choosing the coupling constant $\lambda \sim 10^{-5}$, and using $\Delta T / T \sim \frac{1}{3} \delta \rho / \rho$. The measurements are quoted in terms of a spectral index $n$, with $n=1.1 \pm 0.5$. These measurements are also consistent with the predictions of inflationary models, and with other mechanisms of inhomogeneity generation, such as cosmic strings.

\section{Conclusions}

The results obtained above suggest that the superluminary model could be an attractive alternative to inflation as a solution to the initial value problem in cosmology. Moreover, our picture of the period immediately following the big bang is radically altered from the standard big bang model. The violation of the conservation of energy in the spontaneously broken symmetry phase can provide an explanation for the creation of matter in the beginning of the Universe, an explanation which is not available in the standard FRW model or the inflationary scenario. The model also provides a possible solution the cosmological costant problem.

The quantum fluctuations of the Higgs field, near the phase transition in the velocity of light, produce microwave background density fluctuations, which are frozen in at the horizon. The spectrum of the fluctuations is gaussian and scale invariant with a scalar field coupling constant, $\lambda \sim 10^{-5}$, which is more reasonable in size than the standard value, $\lambda \sim 10^{-14}$, predicted by generic inflationary models [12, 13]. Further work is necessary to investigate in more detail the predictions of the density fluctuation spectrum in the superluminary model.

The superluminary model of the early Universe[1-3] was introduced to provide an interesting alternative to the inflationary model, at a time when the latter model was enjoying a popular revival. It is remarkable that that there has been no serious alternative model considered besides the superluminary model (or varying light speed model, as it is called by Albrecht and Maguiejo (4) . With the advent of a new generation of accurate satellite measurements of the microwave background, it may be possible to distinguish the specific predictions of the two models and the predictions of other alternative models that may be forthcoming in the future.

An important feature of the superluminary model is the unavoidable 
breaking of local Lorentz invariance in the early Universe. In view of the significant changes that will occur in the fundamental physics, it is necessary to have a well defined model for this symmetry breaking. Such a model is provided by the spontaneous symmetry breaking of the Lagrangian in the scenario presented in earlier publications[1-3] and in the present exposition. The "hidden" symmetry of the gravitational vacuum has the advantage, enjoyed in the standard model of particle physics, of retaining the vital features of gauge symmetries, such as Ward identities, in a future theory of quantum gravity.

In recent work [17, 18], gravitational theories based on a bimetric structure formed from a metric and a vector field or the gradient of a scalar field have been proposed. These theories begin with a Lorentz and diffeomorphism invariant formulation and provide an alternative picture to the one described here, based on a rapid change in the velocity of light associated with a phase transition at a critical time $t=t_{c}$ in the early universe when local Lorentz invariance symmetry is broken.

\section{Acknowledgments}

This work was supported by the Natural Sciences and Engineering Research Council of Canada.

\section{References}

[1] J. W. Moffat, Int. J. Mod. Phys. D2, 351 (1993). gr-qc/9211020.

[2] J. W. Moffat, Review Talk given at the International Conference on Problems in Gravitation and Particle Physics, Institute for Theoretical Physics, Protvino, Russia, June 1992.

[3] J. W. Moffat, Found. of Phys. 23, 411 (1993). gr-qc/9209001.

[4] A. Albrecht and J. Maguiejo, astro-ph/9811018.

[5] J. D. Barrow, astro-ph/9811022.

[6] J. D. Barrow and J. Maguiejo, astro-phy/9811072. 
[7] N. Turok and S. W. Hawking, Phys. Lett. B425, 25 (1998); Phys. Lett. B432, 271 (1998).

[8] A. D. Linde, Phys. Rev. D58, 083514 (1998).

[9] A. Vilenkin, Phys. Rev. D58, 083514 (1998)

[10] T. Harko and M. K. Mak, Class. Quantum Grav. 16, 2741 (1999).

[11] M. K. Mak and T. Harko, Class. Quantum Grav. 16 (1999), to be published.

[12] A. H. Guth, Phys. Rev. D23, 347 (1981).

[13] For reviews of inflationary cosmologies, see: E. W. Kolb and M. S. Turner, The Early Universe (Addison-Wesley Pub. Co. California, 1990); A. D. Linde, Particle Physics and Inflationary Cosmology (Harwood, Chur, Switzerland, 1990).

[14] J. M. Bardeen, P. J. Steinhardt and M. S. Turner, Phys. Rev. D28, 679 (1983).

[15] E. R. Harrison, Phys. Rev. D1, 2726 (1970); Ya. B. Zel'dovich, Mon. Not. Roy. Astron. Soc. 160, 1p (1972).

[16] G. F. Smoot et al., Ap. J. Letts. 396, L1 (1992); C. Bennett et al., ibid. 396, L7 (1992).

[17] M. A. Clayton and J. W. Moffat, Phys. Lett. B460, 263 (1999), astroph/9812481.

[18] M. A. Clayton and J. W. Moffat, gr-qc/9910112. 